\title{
Computational screens can speed up the discovery of pharmaceutical cocrystals
}

\author{
Rafel Prohens ${ }^{a}$ and Christopher A. Hunter ${ }^{b}$ \\ ${ }^{a}$ Unitat de Polimorfisme i Calorimetria, Centres Científics i Tecnològics, Universitat de Barcelona, Baldiri Reixac 10, \\ 08028 Barcelona, Spain \\ ${ }^{b}$ Department of Chemistry, University of Cambridge, Lensfield Road, Cambridge CB2 1EW, UK \\ E-mails: rafel@ccit.ub.edu (Prohens); herchelsmith.orgchem@ch.cam.ac.uk (Hunter)
}

Received: November 26, 2018; Accepted: November 27, 2018; Published: December 27, 2018

\begin{abstract}
The calculation of Surface Site Interaction Points for cocrystal computational screens in combination with efficient experimental cocrystallization techniques has been applied successfully to several drug compounds. The basics of this combined approach are briefly reviewed in this communication.
\end{abstract}

\section{Keywords}

Cocrystals; virtual screen; H-bond parameters

The search for new cocrystals of APIs can be very expensive in terms of time and economical resources since the number of potential coformers available with suitable toxicity profiles is huge. Thus, there is a real need in the pharmaceutical industry to optimize screening methodologies, because cocrystals offer solutions to some of the drawbacks inherent to many drug candidates, such as low solubility and bioavailability. Historically, the supramolecular synthon approach has been the paradigm of crystal engineering for the design of new multicomponent crystals based on strong intermolecular interactions [1]. Although very limited in the scope for efficiently locating new coformers, supramolecular synthons are the basis for understanding the formation of a cocrystal. More recently other approaches have been developed for predicting the outcome of cocrystallization experiments. The Hansen solubility parameter [2] has been investigated to guide cocrystal screening. Statistical analyses of the properties of compounds known to form cocrystals show promise. For example, Fabián showed that cocrystals are usually formed by molecules of similar shapes and polarities [3], and the hydrogen bond propensity tool developed by the Cambridge Crystallographic Data Centre has been used to predict the cocrystallization of several APIs [4]. Computational methods have also been applied to the cocrystal problem. A high-level DFT study of 350 organic cocrystals concluded that the formulation of general rules to guide cocrystal formation remains difficult [5].

We have developed a fast computational method to screen large libraries of coformers [6]. The approach uses Hunter's hydrogen bond parameters ( $\alpha$ and $\beta$ ) [7] to predict the formation of intermolecular interactions in crystals and is based on the principle that the crystal structure of an organic compound is 
controlled by a hierarchical organisation of functional group interactions. Thus, the energy of a solid form $(E)$ can be calculated by pairing the best $\mathrm{H}$-bond donor with the best $\mathrm{H}$-bond acceptor, the second best $\mathrm{H}$ bond donor with the second best $\mathrm{H}$-bond acceptor, and so on, until all of the interaction sites $(\mathrm{i}, \mathrm{j})$ are satisfied (Eq. 1).

$$
E=-\sum_{i j} \alpha_{i} \beta_{j}
$$

The difference between the interaction site pairing energies of the cocrystal $\left(E_{\mathrm{cc}}\right)$ and the two components $\left(E_{1}\right.$ and $\left.E_{2}\right)$ is related to the cocrystallization probability (Eq. 2).

$$
\Delta E=E_{c c}-n E_{1}-m E_{2}
$$

where $\mathrm{n}$ and $\mathrm{m}$ refer to the cocrystal stoichiometry.

The method is based on assumptions that ignore all geometrical aspects of crystal packing: (a) all potential interactions in the crystalline solid are established (b) all $\mathrm{H}$-bond sites on the surface of a molecule are independent and free to interact with any other site in the solid, (c) steric constraints on contacts are not considered, (d) cooperativity between sites is ignored. The consequence of these approximations is that the method offers a solution to the problem of rapid virtual screening of large compound libraries. The method was initially calibrated by performing calculations on two APIs with the highest number of reported experimental cocrystals (caffeine and carbamazepine) and a list of nearly 1,000 potential coformers, providing a validation of the relationship between the value of $\Delta \mathrm{E}$ and the probability of cocrystal formation (Fig.1).

(a)

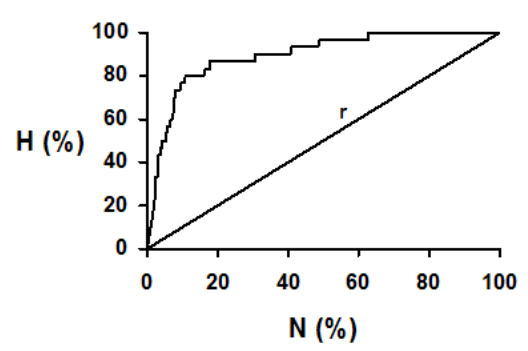

(b)

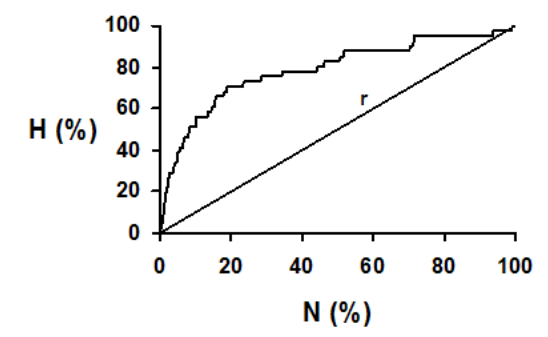

(c)

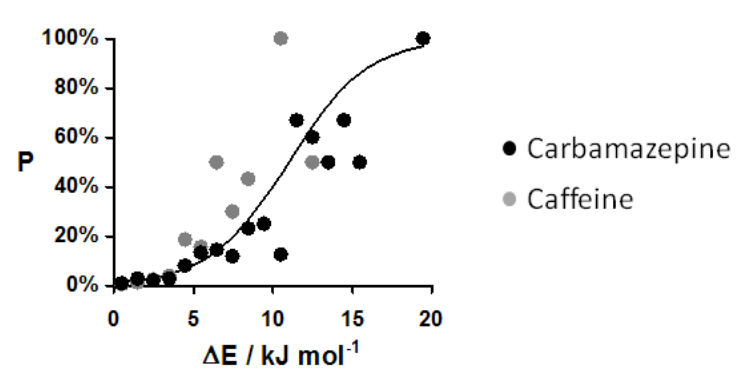

Figure 1. Recall plots for the prediction of 1:1 cocrystals of caffeine (a) and carbamazepine (b) with 849 and 860 potential coformers respectively. $H$ is the fraction of total hits found plotted as function of the fraction of compounds screened $(N)$. The line $(r)$ represents probability of finding a hit as the result of random chance. (c)

Relationship between the probability of obtaining a cocrystal, $P$, and the calculated cocrystal energy, $\Delta E$.

In addition, a set of validations was conducted with cocrystal screens of nine APIs from the literature (diclofenac, piracetam, pyrazine carboxamide, acetazolamide, indomethacin, furosemide, nalidixic acid, 
paracetamol and a drug candidate) that had reported both successful and unsuccessful coformers, and in most cases, calculations reproduced the experimental results [8]. The method has been further applied in combination with experimental screening techniques to optimize the search for new cocrystals with improved physicochemical properties of APIs, such as nalidixic acid [9] spironolactone, griseofulvin [10], zafirlukast [11], sildenafil [12,13] and a novel inhaled JAK-STAT inhibitor [14].

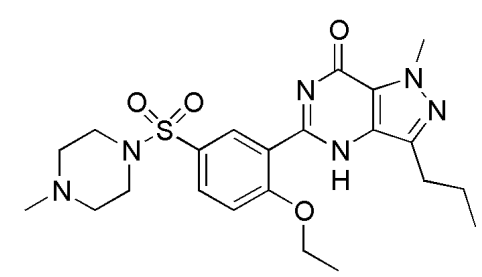

Sildenafil<smiles>CCn1cc(C(=O)O)c(=O)c2ccc(C)nc21</smiles>

Nalidixic acid

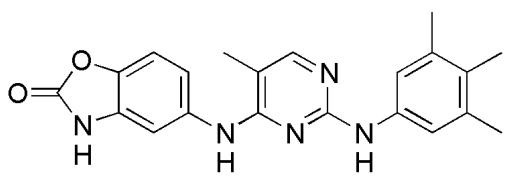

JAK-STAT inhibitor

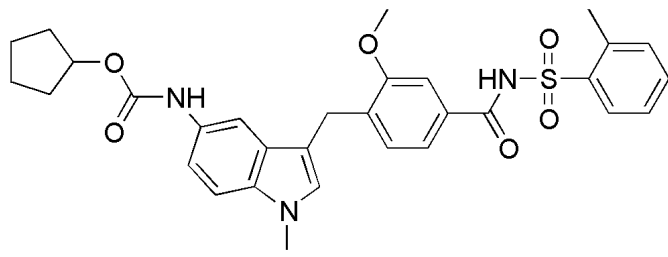

Zafirlukast

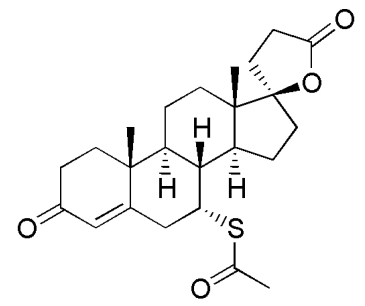

Spironolactone

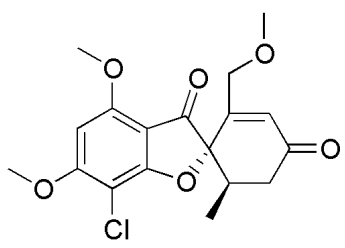

Griseofulvin

Figure 2. Drug compounds tested in combined virtual/experimental cocrystal screens

Virtual cocrystal screening for coformer selection is just part of the more complicated procedure of cocrystal investigation in the pharmaceutical industry. Thus, other pre-screening techniques must be applied prior to the experimental search for new cocrystals in order to reduce time and optimize resources. For instance, solubility prediction can also be conducted if an increase/decrease in solubility is a requirement for the formulation [15], and the safety/toxicity profile of every potential coformer must be assessed using databases or in silico prediction [16]. Finally, once all the computational work is done, information about the solubility of the API and the chosen coformers in the organic solvents used for crystallisation and about the presence of polymorphism/solvatomorphism is necessary. This information is essential for selection of the experimental conditions which will allow the most extensive and efficient exploration of the cocrystal landscape. In summary, the use of computational approaches together with experimental screening methodologies, if combined efficiently, can reduce the time and cost of the development of a new cocrystal formulation.

\section{References}

[1] G.R. Desiraju. Supramolecular Synthons in Crystal Engineering - A New Organic Synthesis. Angew. Chem. Int. Ed. 34 (1995) 2311-2327.

[2] M.A. Mohammad, A. Alhalaweh, S.P. Velaga. Hansen solubility parameter as a tool to predict cocrystal formation. Int. J. Pharm. 407 (2011) 63-71.

[3] L. Fabián, Cambridge Structural Database Analysis of Molecular Complementarity in Cocrystals. Cryst. Growth Des. 9 (2009) 1436-1443.

[4] A. Delori, P.T.A. Galek , E. Pidcock, M. Patni, W. Jones. Knowledge-based hydrogen bond prediction and the synthesis of salts and cocrystals of the anti-malarial drug pyrimethamine with various drug and GRAS molecules. CrystEngComm 15 (2013) 2916-2928. 
[5] C.R. Taylor, G.M. Day. Evaluating the Energetic Driving Force for Cocrystal Formation. Cryst. Growth Des. 18 (2018) 892-904.

[6] D. Musumeci, C.A. Hunter, R. Prohens, S. Scuderi, J.F. McCabe. Virtual cocrystal screening. Chem. Sci. 2 (2011) 883-890.

[7] C.A. Hunter. Quantifying intermolecular interactions: guidelines for the molecular recognition toolbox. Angew. Chem. Int. Ed. 43 (2004) 5310-5324.

[8] T. Grecu, C.A. Hunter, E.J. Gardiner, J.F. McCabe. Validation of a Computational Cocrystal Prediction Tool: Comparison of Virtual and Experimental Cocrystal Screening Results. Cryst. Growth Des. 14 (2014) 165-171.

[9] T. Grecu, H. Adams, C.A. Hunter, J.F. McCabe, A. Portell, R. Prohens. Virtual Screening Identifies New Cocrystals of Nalidixic Acid. Cryst. Growth Des. 14 (2014) 1749-1755.

[10] T. Grecu, R. Prohens, J.F. McCabe, E.J. Carrington, J.S. Wright, L. Brammer, C.A. Hunter. Cocrystals of spironolactone and griseofulvin based on an in silico screening method. CrystEngComm 19 (2017) 3592-3599.

[11] A. Llinas, R. Barbas, M. Font-Bardia, M. J. Quayle, S. Velaga, R. Prohens. Two New Polymorphic Cocrystals of Zafirlukast: Preparation, Crystal Structure, and Stability Relations. Cryst. Growth Des. 15 (2015) 4162-4169.

[12] P. Lawton, H. L. Jones, N.M. Kanji, R. Barbas, R. Prohens. PCT/GB2017/050921.

[13] R. Barbas, M. Font-Bardia, A. Paradkar, C.A. Hunter, R. Prohens. Combined Virtual/Experimental Multicomponent Solid Forms Screening of Sildenafil: New Salts, Cocrystals, and Hybrid SaltCocrystals. Cryst. Growth Des. (2018) In Press: doi: 10.1021/acs.cgd.8b01413.

[14] A. Llinas, R. Barbas, M. Font-Bardia, A. Smailagic, R. Prohens. Derisking development by cocrystallisation screen of a novel selective inhaled JAK-STAT inhibitor. Cryst. Growth Des. (2018) In Press: doi: 10.1021/acs.cgd.8b01492.

[15] C.A. Hunter, R. Prohens. Solid form and solubility. CrystEngComm 19 (2017) 23-26.

[16] P.A. Corner, D. Berry, J.F. McCabe, R. Barbas, R. Prohens, H. Du, H. Zhou, A. Llinas. Property prediction and pharmacokinetic evaluation of mixed stoichiometry cocrystals of zafirlukast, a drug delivery case study. CrystEngComm 20 (2018) 1346-1351.

(C)2018 by the authors; licensee IAPC, Zagreb, Croatia. This article is an open-access article distributed under the terms and conditions of the Creative Commons Attribution license (http://creativecommons.org/licenses/by/3.0/) (cc) EY 